\title{
Monopolos Circular e Elíptico de Microfita para Sistemas UWB
}

\author{
Marcelo Ribeiro da Silva, Clarissa de Lucena Nóbrega, Angela Machado Zenaide, \\ Paulo Henrique da Fonseca Silva e Adaildo Gomes D'Assunção
}

\begin{abstract}
Resumo-Este trabalho apresenta uma comparação entre as propriedades de monopolos de microfita para aplicações em sistemas de banda ultralarga (ultra wideband, UWB). São consideradas duas configurações de monopolos elípticos, com excentricidades diferentes, e uma de monopolo circular. São construídos dois protótipos para cada configuração de antena, sendo um com a configuração típica de microfita e outro similar ao primeiro, mas com uma pequena abertura no plano de terra. Os resultados medidos mostram que a introdução da abertura retangular no plano de terra permite melhorar a resposta em freqüência das antenas consideradas. Observa-se concordância entre os resultados medidos e simulados.
\end{abstract}

Palavras-chave-Antena de microfita, monopolo de microfita, patch elíptico, patch circular, banda ultralarga, UWB.

\begin{abstract}
This work presents a comparison among the properties of three microstrip monopoles for ultra wideband (UWB) systems. Two microstrip elliptical monopoles, with different values of the excentricity, and one microstrip circular monopole are investigated. Two prototypes are built for each one of these microstrip antennas, the first with the basic microstrip monopole configuration and the second similar to the first one but with a slit on the ground plane. Measured results show that the presence of the slit on the monopole ground plane helps to improve the performance of these antennas. Measured and simulated results are in agreement.
\end{abstract}

Keywords-Microstrip antenna, microstrip monopole, elliptical patch, circular patch, ultra wideband, UWB.

\section{INTRODUÇÃO}

As antenas planares, como as antenas de microfita, são muito utilizadas nos sistemas de comunicações sem fio, o que justifica o grande interesse de muitos pesquisadores nas duas últimas décadas [1]. No último ano, uma atenção muito especial foi dada ao desenvolvimento de estudos de antenas UWB. [2]-[5], para aplicações na faixa de 3,1 a 10,6 GHz. Em particular, foram investigadas as propriedades de antenas monopolos de microfita, sendo propostos diversos formatos para os patches condutores dessas antenas [2]-[5].

Esses monopolos de patches condutores são, em geral, construídos em estruturas de microfita com planos de terra limitados, ou truncados, com a remoção do cobre se dando de forma perpendicular às linhas de alimentação, que são introduzidas nos planos dos patches, o que simplifica consideravelmente o processo de fabricação. Em trabalhos recentes, a análise dos parâmetros principais dessas antenas tem sido efetuada através de softwares comerciais, como o Ansoft HFSS [2], [3].

Marcelo Ribeiro da Silva, marcelo cefetpb@hotmail.com, Clarissa de Lucena Nóbrega, clarissalucen@hotmail.com, Ângela Machado Zenaide, angelazenaide@gmail.com, Adaildo G. D’Assunção, adaildo@ct.ufrn.br, Universidade Federal do Rio Grande do Norte, Departamento de Engenharia Elétrica, Natal, RN, Tel: +55-84-3215-3731. Paulo H. da Fonseca Silva, henrique@cefetpb.edu.br, CEFET-PB, GTEMA, João Pessoa, PB, Tel.: +55-83-3208-3055. Este trabalho foi parcialmente financiado pelo CNPq e pela CAPES.
Neste trabalho, efetua-se uma investigação teórica e experimental das propriedades dos monopolos de microfita de patches circulares e elípticos, para aplicações em sistemas UWB. A estrutura apresentada em [2], com a geometria mostrada na Fig. 1, foi considerada como referência para a realização da investigação experimental, realizada com a construção e a medição de vários protótipos, enquanto o Ansoft Designer foi usado como ferramenta auxiliar no processo de simulação.

O objetivo principal é modificar a configuração do plano de terra de monopolos de patches circulares e elípticos, de forma a melhorar os seus desempenhos. Como mostrado nas Figs. 2 e 3, aos truncamentos dos planos de terra, que são representados por cortes lineares, acrescenta-se também a remoção de uma pequena porção do plano de terra (recorte retangular) na região abaixo da linha de microfita de alimentação, como proposto em [5].

São mostradas curvas da perda de retorno e do SWR em função da freqüência, para antenas de microfita com patches circulares e elípticos em estruturas com o plano de terra truncado e com o plano de terra otimizado (truncado e com o recorte na borda), como mostrado nas Figs. 2.b e 3.b.

\section{GEOMETRIAS CONSIDERADAS}

As configurações de antenas de microfita consideradas neste trabalho estão mostradas nas Figs. 2 e 3 . Como antenas de microfita, elas são constituídas por patches condutores, com formatos elípticos (Fig. 2), ou com formatos circulares (Fig. 3), depositados sobre substratos dielétricos, que se encontram apoiados sobre planos de terra. São consideradas duas estruturas principais, que são denominadas de acordo com o formato do plano de terra. Nas Figs. 2.a e 2.b, as fotos situadas à esquerda, mostram a estrutura com plano de terra truncado (monopolo), enquanto que as fotos localizadas à direita, exemplificam o caso em que o plano de terra truncado é otimizado em relação à largura de banda, aumentada para as antenas com a introdução de um recorte retangular. As espessuras dos patches condutores e as perdas dielétricas e condutoras são desprezadas. O substrato dielétrico é considerado isotrópico, com permissividade elétrica relativa, $\varepsilon_{\mathrm{r}}$, e espessura, t.

$\mathrm{O}$ patch condutor elíptico tem um comprimento $a$ e uma largura $b$, definidos como indicado na Fig. 1. Esse patch é alimentado por uma linha de microfita de $50 \Omega$, de comprimento $l$, introduzida no mesmo plano do patch, o que simplifica a sua construção e se constitui em uma escolha típica. Para analisar o patch condutor circular, considera-se $a=b$. Outras dimensões importantes nas estruturas consideradas são: a largura da microfita de alimentação, $\mathrm{W}_{0}$, e a distância horizontal, $g$, do ponto de conexão da microfita com o patch elíptico ao plano vertical de indicação do 
truncamento do plano de terra da microfita. As dimensões do recorte retangular $\alpha$ e $\beta$, são medidas, respectivamente, ao longo do truncamento do plano de terra e na direção da linha de microfita (Figs. 2.b e 3.b, fotos da direita).

\section{Projeto, Construção e Resultados}

$\mathrm{Na}$ investigação experimental, foram usadas placas de fibra de vidro cobreadas nas duas faces. A espessura da camada dielétrica, $\mathrm{t}$, é $1,587 \mathrm{~mm}$ e a permissividade elétrica relativa, $\varepsilon_{\mathrm{r}}$, é 4,4 .

A razão entre os comprimentos $b$ e $a$ da elipse (Fig. 1) foi considerada igual a 0,375 e 0,75 . Estes valores foram considerados para facilitar comparações com os resultados dos casos particulares apresentados em [3].

Foram construídos e medidos vários protótipos com os mesmos comprimentos da elipse e o mesmo substrato dielétrico. No entanto, os valores das dimensões $\alpha$ e $\beta$ dos recortes retangulares introduzidos nos planos de terra (Figs. 2.b e 3.b, fotos da direita) foram variados no processo de otimização.

Nas medições, foi usado um analisador de circuitos para a obtenção da perda de retorno e do SWR em função da freqüência.

A Fig. 4 mostra os resultados obtidos nas medições para a perda de retorno e o SWR na entrada da antena monopolo de microfita com patch circular (plano de terra truncado e sem recorte) para freqüências de $300 \mathrm{kHz}$ a $13,51 \mathrm{GHz}$. Os parâmetros estruturais principais são $b / a=1, a=b=60$ $\mathrm{mm}, \mathrm{W}=60 \mathrm{~mm}, \mathrm{~L}=101 \mathrm{~mm}, \mathrm{~W}_{0}=3 \mathrm{~mm}, \mathrm{t}=1,587 \mathrm{~mm} \mathrm{e}$ $\varepsilon_{\mathrm{r}}=4,4$.

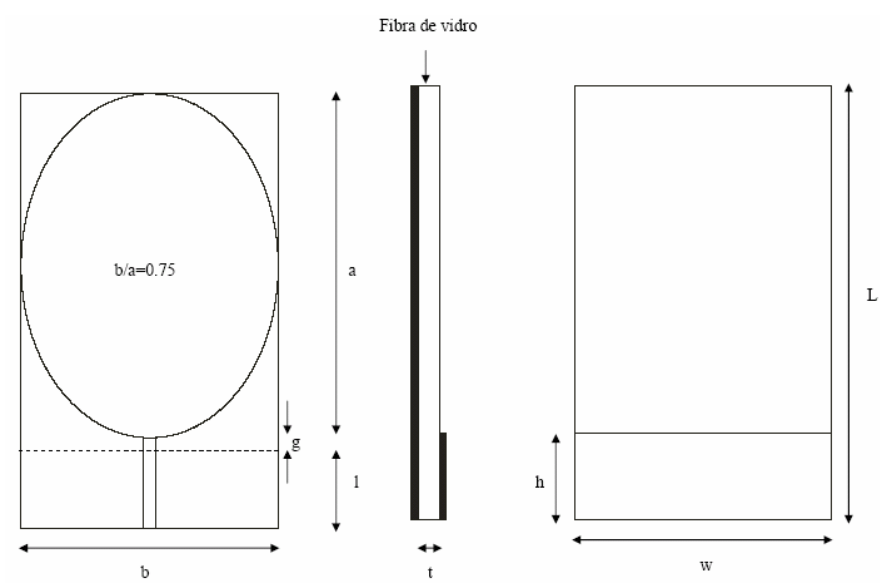

Fig. 1. Geometria de monopolo de microfita com patch elíptico.

$\mathrm{Na}$ Fig. 4.a, os resultados medidos mostram que o monopolo de patch circular com o plano de terra truncado (Fig. 3.b, foto da esquerda) apresenta níveis de perda de retorno oscilando em torno de $-15 \mathrm{~dB}$ na maior parte da faixa de freqüência considerada. Na Fig. 4.b, os resultados medidos para o SWR na faixa de freqüência de 3,1 a 10,6 $\mathrm{GHz}$, confirmam que este monopolo apresenta uma boa resposta $(\mathrm{SWR} \leq 2)$ para utilização em sistemas UWB.

A Fig. 5 mostra os resultados medidos para o monopolo de patch circular com recorte no plano de terra truncado (Fig. 3.b, foto da direita).

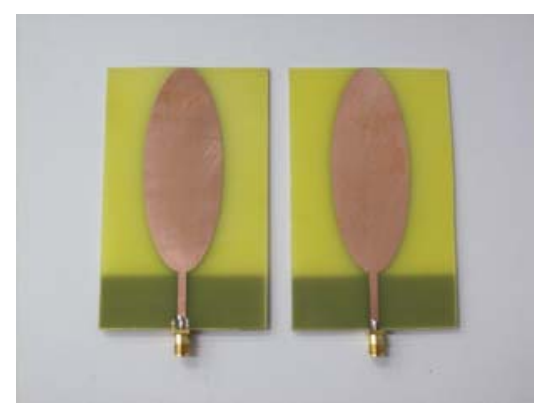

(a) Vista superior.

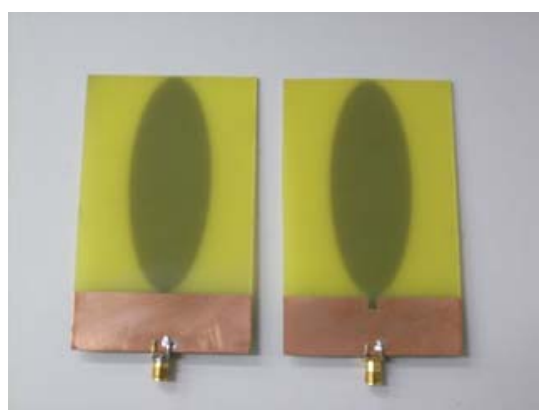

(b) Vista de baixo

Fig. 2. Monopolos de microfita com patches elípticos e diferentes configurações do plano de terra. As fotos destacam: (a) os patches condutores e (b) os planos de terra.

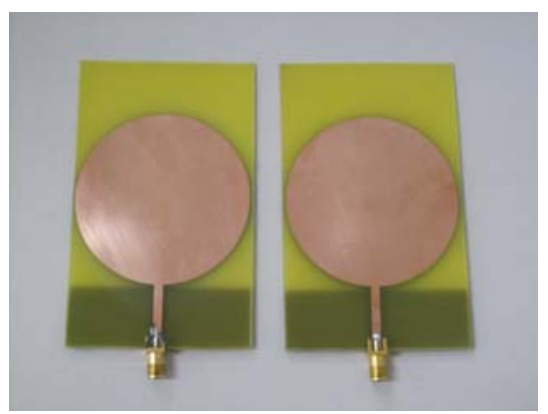

(a) Vista superior.

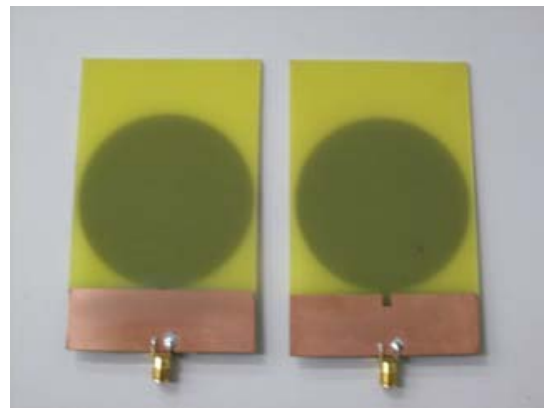

(b) Vista de baixo.

Fig. 3. Monopolos de microfita com patches circulares e diferentes configurações do plano de terra. As fotos destacam: (a) os patches condutores e (b) os planos de terra.

Observe que a introdução do recorte retangular, que apresenta dimensões $\alpha=3 \mathrm{~mm}$ e $\beta=4 \mathrm{~mm}$, melhorou a resposta em freqüência desta antena em relação à da antena sem recorte no plano de terra (Fig. 3.b, foto da esquerda), tanto para a perda de retorno, com valores oscilando em torno de $-20 \mathrm{~dB}$, como para o SWR, que apresenta valores nitidamente menores. 


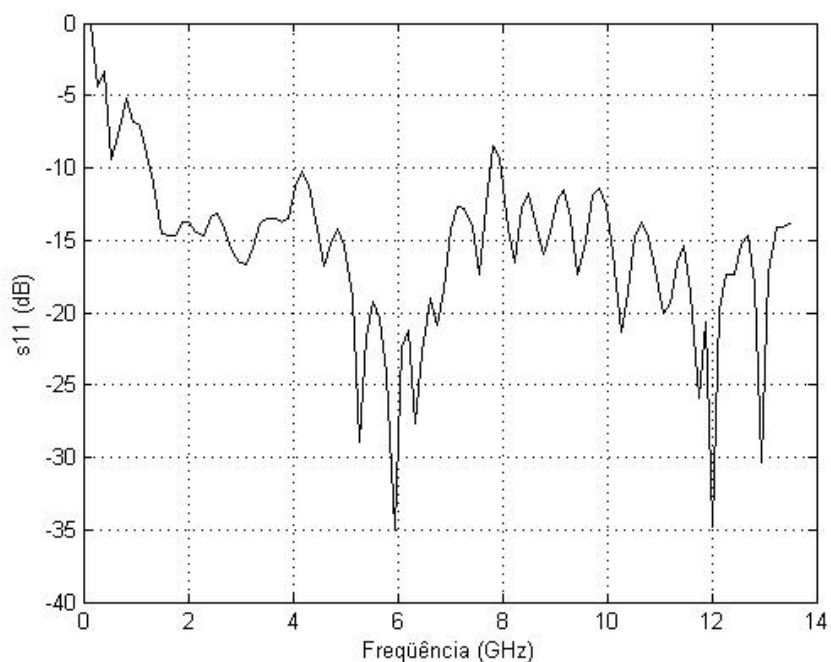

(a) Perda de retorno.

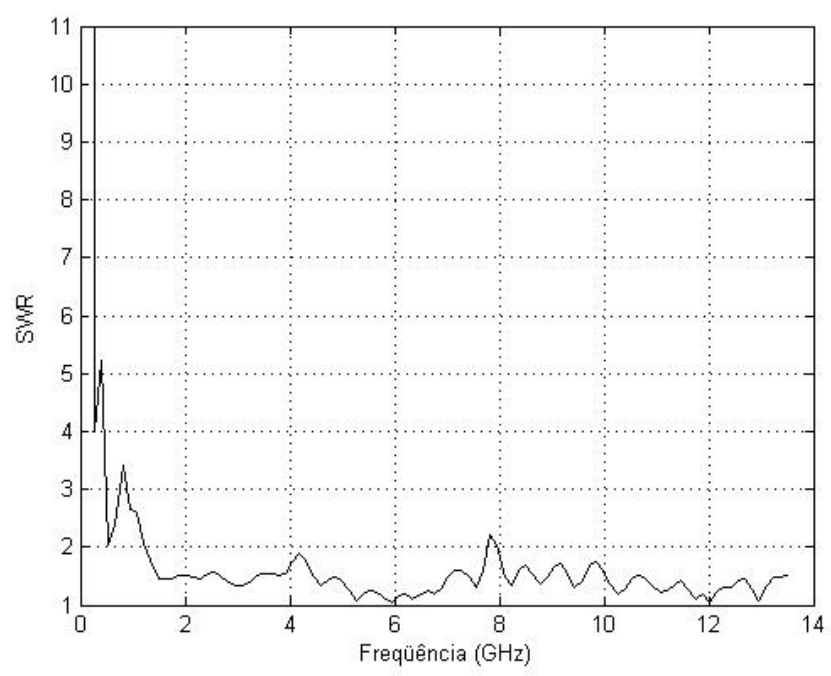

(b) SWR.

Fig. 4. Resultados medidos para a antena de patch circular de microfita, com plano de terra truncado. Parâmetros estruturais: $a=b=60 \mathrm{~mm}, g=1$ $\mathrm{mm}, l=20 \mathrm{~mm}, \mathrm{~W}=60 \mathrm{~mm}, \mathrm{~L}=101 \mathrm{~mm}, \mathrm{~W}_{0}=3 \mathrm{~mm}, \mathrm{t}=1,587 \mathrm{~mm}$ e $\varepsilon_{\mathrm{r}}=$ 4,4. Faixa de freqüência: $300 \mathrm{kHz}$ a $13,5 \mathrm{GHz}$.

A Fig. 6 mostra uma comparação entre os resultados medidos para as antenas: (a) patch elíptico (Fig. 2), com $b / a=0,75$, (b) patch elíptico (Fig. 2), com $b / a=0,375$ e (c) patch circular (Fig. 3), nos casos sem e com recorte retangular nos seus planos de terra. São apresentados resultados medidos para o SWR em função da freqüência, na faixa de $300 \mathrm{kHz}$ a $13,5 \mathrm{GHz}$.

Das estruturas consideradas a que apresenta melhores resultados é a do patch circular com recorte no plano de terra (Fig. 3, foto da direita). As estruturas com patches elípticos sem compensação no plano de terra são as que apresentam os piores desempenhos. Observa-se, no entanto, que a introdução do recorte nos planos de terra permite melhorar de forma acentuada essas respostas. São observados valores inferiores a 2 na faixa de freqüências de interesse, exceto para o monopolo elíptico com $b / a=0,375$. Para esta antena, o valor máximo do SWR é inferior a 2,3.

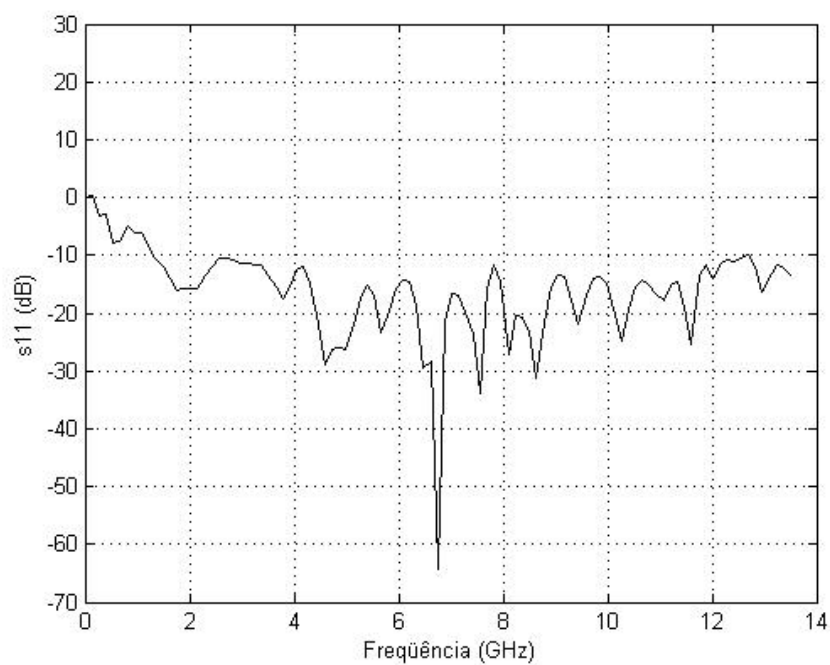

(a) Perda de retorno.

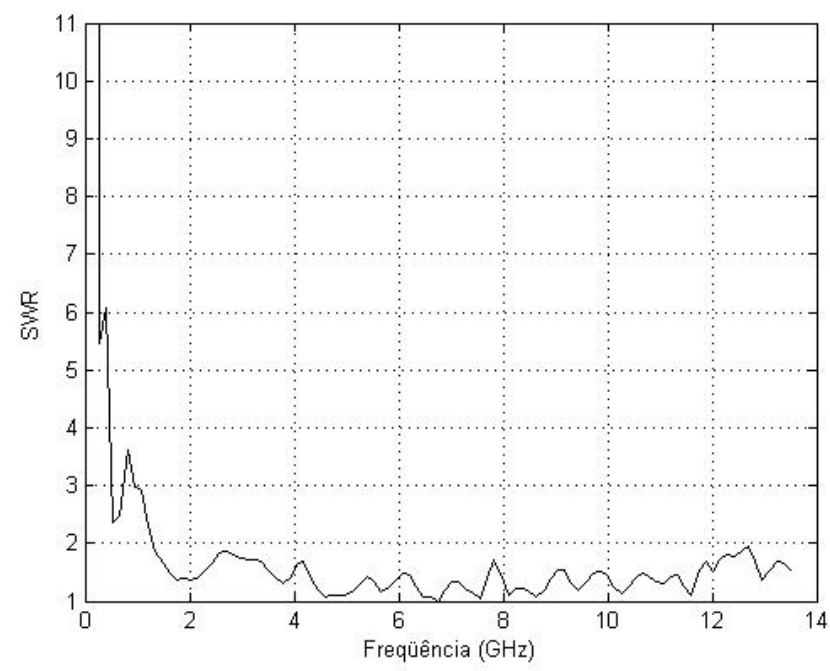

(b) SWR.

Fig. 5. Resultados medidos para a antena de patch circular de microfita com recorte no plano de terra. Parâmetros estruturais: $a=b=60 \mathrm{~mm}, g=1 \mathrm{~mm}$, $l=20 \mathrm{~mm}, \mathrm{~W}=60 \mathrm{~mm}, \mathrm{~L}=101 \mathrm{~mm}, \mathrm{~W}_{0}=3 \mathrm{~mm}, \mathrm{t}=1,587 \mathrm{~mm}, \alpha=3 \mathrm{~mm}$, $\beta=4 \mathrm{~mm}$ e $\varepsilon_{\mathrm{r}}=4,4$. Faixa de freqüência: $300 \mathrm{kHz}$ a $13,5 \mathrm{GHz}$.

\section{CONClusÃO}

Foram apresentados os resultados obtidos em uma investigação experimental das propriedades de monopolos de microfita com patches circulares e elípticos sobre substratos dielétricos isotrópicos. Vários protótipos foram construídos e medidos. Os efeitos produzidos por modificações efetuadas no plano de terra (um recorte retangular), tanto na perda de retorno como no SWR, na entrada de cada antena, foram investigados. Os resultados obtidos mostraram a importância da otimização do formato do plano de terra, que propicia a redução da capacitância da linha de microfita na região de conexão com o patch condutor, para fins de casamento de impedâncias. Na região de aplicações UWB, o SWR medido é inferior a 1,6 para o monopolo patch circular compensado. As estruturas compensadas permitiram obter excelentes larguras de banda (maiores que 20:1), quando calculadas para uma perda de retorno inferior a $-10 \mathrm{~dB}$, sendo adequadas para utilização nos sistemas atuais de comunicações sem fio. 


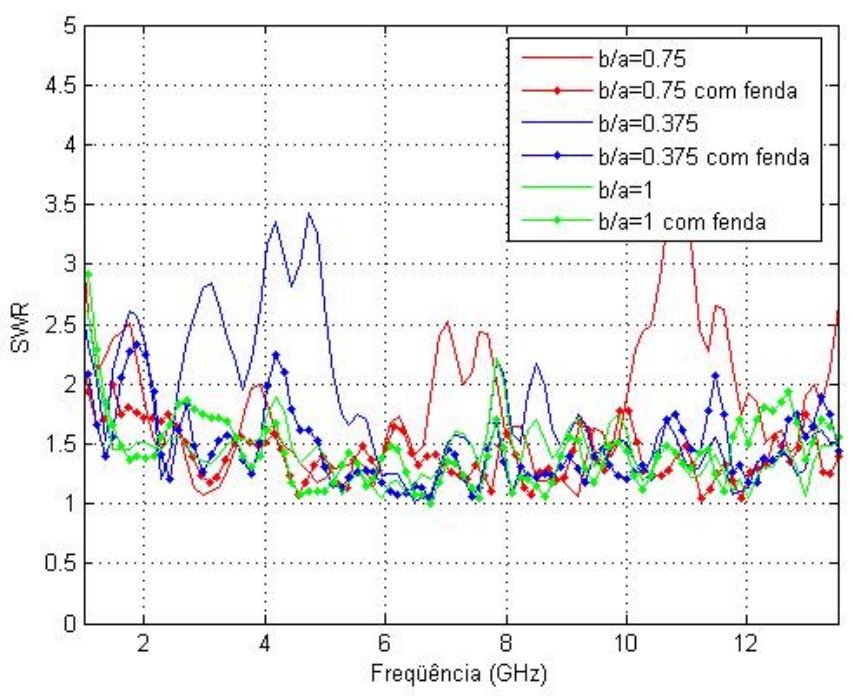

Fig. 6. Resultados medidos para o SWR dos monopolos circulares e elípticos, com e sem recorte no plano de terra, para $a=80 \mathrm{~mm}, g=1 \mathrm{~mm}$, $l=20 \mathrm{~mm}, \mathrm{~W}=60 \mathrm{~mm}, \mathrm{~L}=101 \mathrm{~mm}, \mathrm{~W}_{0}=3 \mathrm{~mm}, \mathrm{t}=1,587 \mathrm{~mm}, \alpha=3 \mathrm{~mm}$, $\beta=4 \mathrm{~mm}$ e $\varepsilon_{\mathrm{r}}=4,4$.

\section{REFERÊNCIAS}

[1] C. A. Balanis, Antenna Theory: Analysis and Design, $3^{\text {a }}$. Ed., Wiley Interscience, 2005.

[2] K. G. Thomas e N. Lenin, "Ultra Wideband Printed Monopole Antenna", Microwave Opt. Technol Lett, vol. 49, pp. 1082-1084, Maio 2007.

[3] C.-H. Hsu, "Planar Multilateral Disc Monopole Antenna for UWB Application", Microwave Opt. Technol Lett, vol. 49, pp. 1101-1103, Maio 2007.

[4] Q. Wu, R. Jin, J. Geng e M. Ding, "Compact CPW-Fed StackedCircle Monopole Antenna with Very Wide Bandwidth", Microwave Opt. Technol Lett, vol. 49, pp. 1192-1194, Maio 2007.

[5] X. L. Bao e M. J. Ammann, "Investigation on UWB Printed Monopole Antenna with Rectangular Slitted Groundplane", Microwave Opt. Technol Lett, vol. 49, pp. 1585-1587, Julho 2007. 